$$
\begin{array}{r}
\text { Entornos } \\
\text { educativos y } \\
\text { agencia de } \\
\text { los escritores }
\end{array}
$$




\title{
Enunciación
}

\section{Identidades profesionales y escritura de narraciones (auto)biográficas en el ingreso a la universidad}

\author{
Professional identities and writing of (auto)biographical narratives \\ when entering the university
}

\author{
${\text { Gustavo Bombini* }{ }^{*} \text {, Paola Iturrioz }}^{* *}$
}

\section{Resumen}

En este trabajo abordamos la cuestión de la producción de identidades profesionales en alumnos ingresantes a la carrera de Profesorado en Letras de la Universidad Nacional de San Martín (Argentina) a partir de un corpus de escritos producidos como actividad final de uno de los módulos del curso de ingreso $(2018,2019,2020)$. La entrada a la escritura en el ámbito académico a partir de textos que reconocen un sesgo autobiográfico se propone como habilitante de las prácticas de la escritura a la hora de poner en cuestión la mirada deficitaria sobre las prácticas letradas de los ingresantes universitarios (Bombini y Labeur, 2017). La perspectiva metodológica elegida en virtud de su productividad para reconocer las estrechas articulaciones entre teoría y práctica es la que ofrece la etnografía de la educación que, en la línea de la investigadora mexicana Elsie Rockwell (2009, 2018) y el equipo del Cinvestav, ha tomado en algunos casos como objeto de análisis la cultura escrita y sus campos de circulación, especialmente las instituciones educativas.

De la lectura de los textos surgieron dimensiones de análisis referidas a las socializaciones familiares, barriales y comunitarias; a las experiencias culturales y escolares previas; a representaciones que anticipan ideas y valoraciones acerca del ámbito académico; elecciones estéticas diversas; núcleos de intereses; fantasías de intervención para la transformación en los campos educativo, cultural, político y lingüístico-literario, y de manera más específica, expectativas acerca de los contenidos de la formación del Profesorado en Letras.

Palabras clave: identidad, escritura, ingreso universitario, etnografía.

\begin{abstract}
In this work we address the question of the production of professional identities in students entering the career of Language and Literature at the National University of San Martín (Argentina) from a corpus of writings produced as the final activity of one of the modules of the course income $(2018,19,20)$. The entry to writing in the academic field from text that recognize an autobiographical bias are proposed as enablers of writing practices when questioning the deficient view of the legal practices of university entrants. (Bombini, Labeur, 2017)

The perspective of analysis chosen by virtue of its productivity to recognize the close articulations between theory and practice, is the one offered by ethnography of education that in the line of the Mexican researcher Elsie Rockwell and the CINVESTAV team (Rockwell, 2009, 2018) has in some cases taken as an object of analysis the written culture and its fields of circulation, especially educational institutions.
\end{abstract}

\footnotetext{
* Doctor en Letras (UBA). Profesor e investigador en la Universidad de Buenos Aires y en la Universidad Nacional de San Martín. Áreas de interés: didáctica de la lengua y la literatura; formación de profesores; literatura infantil. Correo electrónico: gbombini@gmail.com

** Profesora en Letras (UNLP). Profesora e investigadora en la Universidad Nacional de San Martín. Áreas de interés: didáctica de la lengua; formación de profesores, educación intercultural bilingüe. Correo electrónico: paolaiturrioz@hotmail.com
}

Cómo citar: Bombini, G., e Iturrioz, P. (2021). Identidades profesionales y escritura de narraciones (auto)biográficas en el ingreso a la universidad. Enunciación, 26, 121-135. DOI: https://doi.org/10.14483/22486798.16380

Artículo postulado: 22 de mayo de 2020; aprobado: 05 de noviembre de 2020 
From the reading of the texts, dimensions of analysis emerged referring to family, neighborhood and community socializations, previous cultural and school experiences, representations that anticipate ideas and valuations about the academic field, diverse aesthetic choices, nucleus of interests, fantasies of intervention for transformation in the educational, cultural, political and linguistic-literary fields and, more specifically, expectations about the content of teacher education in language and literature.

Keywords: identity, writing, university entrance, ethnography.

\section{Introducción}

Como parte de los requisitos iniciales para el acceso a carreras de grado de las universidades públicas argentinas, algunas de esas casas de estudio, a través de sus facultades, institutos o escuelas, establecen la obligatoriedad del cumplimiento de un curso de ingreso o curso preparatorio que asume diferentes modalidades y formatos para su realización.

Como dato significativo para comprender el sentido de este espacio de formación inicial, es preciso señalar que la universidad pública argentina, así como también la educación superior terciaria, reconoce una tradición construida a lo largo del siglo XX sustentada en la idea de la educación entendida como un derecho, por ende, libre y gratuita y sin restricciones para su ingreso. Las políticas universitarias de las dos primeras décadas de este siglo reforzaron aquella tradición y, a partir de la creación de nuevas casas de estudio ubicadas en variedad de territorios de provincias y del conurbano de la Ciudad de Buenos Aires, generaron de este modo una estrategia de diversificación y descentralización de la oferta académica respecto de la antigua supremacía de las universidades tradicionales.

La Universidad Nacional de San Martín, y su Escuela de Humanidades, pertenece al grupo de universidades de la denominada área metropolitana y está ubicada en el partido del mismo nombre. Se trata de una zona con fuerte desarrollo industrial con algunos sectores de pobreza y marginalidad. En este contexto muchos estudiantes que asisten a la universidad provienen de estos sectores desfavorecidos y, en la mayoría de los casos, son primera generación de estudiantes secundarios y universitarios de sus familias. Muchos de ellos no se dedican únicamente a estudiar, sino que desarrollan tareas dentro de la economía informal.

En el caso de la Escuela de Humanidades de la Universidad Nacional de San Martín, el requisito de ingreso se denomina "Curso preparatorio universitario", conocido como CPU, y está dirigido a aspirantes a las carreras de profesorado y licenciatura en Historia, Letras, Filosofía, Ciencias de la Educación, Psicopedagogía y Comunicación. El CPU consta de tres módulos denominados: "Introducción a los estudios universitarios", "Taller de lectura y escritura" y "Módulo específico".

\section{Marco teórico o conceptual}

Esta investigación se enmarca dentro de la perspectiva de los nuevos estudios de literacidad, especialmente a partir del concepto de prácticas letradas utilizado por Virginia Zavala (2009), para dar cuenta de las relaciones entre las actividades y los textos, para realizar una comprensión simultánea de las esferas individuales y sociales, con el propósito de observar los procesos internos del sujeto y los procesos sociales que incluyen saberes compartidos representados en ideologías e identidades sociales. Interesa también la recuperación de la perspectiva bajtiniana en torno a la producción de los géneros discursivos en aras de reconocer el carácter histórico y cultural, así como también la variedad de estos géneros por cuanto dependen de cada esfera de uso y de los estilos personales y repertorios particulares. Desde este marco, la especialista en etnografía de la educación Elsie Rockwell (2018) denomina "géneros de enseñanza" (p. 575) al resultado de una combinación particular de géneros, múltiples y cambiantes que, en nuestro caso, asumen las particularidades y las necesidades didáctico-pedagógicas del tramo de la zona de pasaje habitualmente llamado ingreso. También, en el marco de la etnografía de la 
educación y de las prácticas de lectura y escritura, se suman las nociones de acceso y apropiación en eventos de lectura y escritura, entendidos como unidades de análisis (Kalman, 2003).

Asimismo, dentro de los estudios dedicados a la escritura, en vinculación con la escritura académica, hemos recuperado el trabajo de Theresa Lillis (2013) quien desde una perspectiva sociolingüística se centra en los procesos de construcción de identidades en la escritura.

Consideramos el análisis de las prácticas de literacidad desde una perspectiva sociocultural como la que aquí asumimos, o en términos de la perspectiva ecológica de autoras como Satcwell e Ivanič (2010) que considera la lectura y la escritura no como un conjunto de habilidades descontextualizadas, sino, como,

[...] an 'ecological' view, in which literacy practices vary from one context to another according to the purposes they serve, according to the meanings and values attached to them, and according to other aspects of their social context. This view leads the researcher to focus on the social, affective, technological and practical. (p. 50)

A su vez, forma parte del marco teórico la idea de creación narrativa del yo del psicólogo cultural Jerome Bruner (2002), abonando a la construcción interdisciplinaria necesaria para el análisis de las prácticas de escritura de los ingresantes que son interpelados en relación con sus expectativas profesionales.

\section{Metodología}

En este trabajo abordaremos un corpus de 100 producciones escritas por estudiantes ingresantes en el Módulo específico del Profesorado Universitario en Letras de las cohortes 2018, 2019 y 2020. La perspectiva de análisis, elegida en virtud de su productividad para reconocer las estrechas articulaciones entre teoría y práctica, es la que ofrece la etnografía de la educación que en la línea de la investigadora mexicana Elsie Rockwell (2009, 2018) y el equipo del Cinvestav ha tomado en algunos casos como objeto de análisis a la cultura escrita y sus campos de circulación, especialmente las instituciones educativas. Así, nos interesan, dentro de esta perspectiva, aquellos recorridos que se centran en el análisis de las prácticas de lectura y escritura situadas y que, por tanto, reconocen las particularidades de las prácticas de los sujetos, los escenarios singulares en los que estas prácticas se desarrollan y las especificidades discursivas, en esferas de uso diferenciadas que, para nuestro caso, son las que se desarrollan en los inicios de la escolarización universitaria.

Tratándose del análisis de un corpus concreto, recuperaremos categorías de análisis etnográficos previos (Kalman, 2003) y otras que emergen del propio trabajo de campo constituyéndose así nuevo conocimiento acerca de la situación de ingreso, de los sujetos ingresantes y de su producción discursiva. A su vez, esta producción de investigación asume una posición de intervención respecto de la propia práctica que analiza. Como docentes del módulo, la interpretación de las producciones de los estudiantes nos brinda elementos que permiten ensayar distintos caminos para la propuesta de enseñanza, a sabiendas de las conocidas tensiones entre producción de investigación y posible impacto de esta en la práctica universitaria (Rockwell, 2009).

En función de la continuidad de este trabajo de investigación, que abordará en su versión final la totalidad del recorrido de formación y las experiencias como profesionales noveles de este grupo de ingresantes, metodológicamente vale la pena recuperar los once aspectos a considerar en el análisis de las diferentes prácticas sociales en general y de las prácticas sociales de literacidad, en particular, propuestas por Satchwell e Ivanič (2010) tal como se presentan en la siguiente cita:

The list of aspects was arrived at through examining the differences between literacy practices in and out of college, as part of the curriculum and as part 
of everyday life, and how each practice was viewed by participants (or partial or non-participants) in that literacy practice in relation to themselves. The list of aspects emerged as common factors that allowed for comparison among practices in the data. For the purposes of this chapter, we are focusing on the following eleven aspects of a literacy practice identited during the project: participants, audience, purpose, text-type, artefact, medium, mode, content, activity, place, and time/duration. Of these, six are aspects of any social practice: participants, purpose, artefact, activity, place, and time/duration and can be mapped onto theoretical representations of the structure of any social practice or of social action, such as the Activity Theory triangle. The others, audience, text-type, medium, mode, and content are specific to communicative practices (spoken, written and visual), and we have found it useful to specify them when analysing and comparing the literacy practices which are the focus of the LfLFE project (Literacies for Learning in Further Education). (p. 50)

Como se verá, en esta etapa inicial se han considerado específicamente los aspectos propios de las prácticas comunicativas (en especial la lectura, la escritura y las interacciones en el aula) que, en una etapa posterior, se triangularán para su análisis con aquellos aspectos propuestos por las autoras citadas como propios de toda práctica social.

\section{Resultados y análisis}

Abordaremos en este artículo el caso del Módulo específico para la carrera de Profesorado Universitario en Letras que se cursa durante seis semanas, entre la primera de febrero y la tercera de marzo, en el momento previo al inicio del calendario académico de cada ciclo lectivo. En clases presenciales (apoyadas en un aula virtual) de tres horas semanales, se les ofrece a los estudiantes una antología preparada ad hoc por los profesores responsables del módulo (autores de este artículo) que incluye textos de diversos géneros, ficcionales y no ficcionales, autobiográficos, algunos de ellos, referidos a experiencias profesionales en el campo de las letras tanto en la investigación, la crítica, la formación docente y la enseñanza, de autores de distintas procedencias y de distinto de grado de circulación y de legitimidad.

Dentro de los textos ficcionales, se eligen algunos que tematizan, mediante el recurso de la parodia, escenas de la vida escolar o profesional. Se trata de un corpus de textos que dista de los criterios de selección habituales para el caso de los cursos introductorios más centrados en textos conceptuales, en registros académicos, como artículos o papers propios de la formación. Ponemos en duda que el trabajo focalizado en los denominados géneros académicos sea la clave del éxito en un curso de ingreso o de nivelación, en principio porque la propia definición de género es controvertida (no guían los mismos patrones de escritura de una monografía sobre el último modelo de lingüística chomskiana que una acerca de las características del barroco en El Quijote, de Cervantes).

También importa destacar que partimos de una posición pedagógica que no se centra en una mirada deficitaria sobre los ingresantes, sino que reconoce la diversidad de experiencias previas y la productividad de tenerlas en cuenta como punto de partida; asimismo dentro de esta posición, se reconoce a la escritura como un práctica sociocultural y de alto impacto en los procesos de subjetivación por lo que, desde las estrategias didácticas, se elaboran recorridos que parten de considerar complejas situaciones de apropiación para primeras prácticas de escritura en un ámbito nunca antes transitado, donde aflojar la mano suele ser el paso inicial necesario antes de abordar cuestiones técnico-retóricas y disciplinares de mayor especificidad (Bombini e Iturrioz, 2017).

Lo dicho supone un distanciamiento de programas de fuerte presencia en la Argentina y en Latinoamérica conocidos como alfabetización académica que, si bien tienen como virtud haber instalado las consideraciones sobre la lectura y la escritura en la agenda de la pedagogía universitaria, han acotado sus planteamientos y propuestas a 
un repertorio cristalizado de géneros legitimados y fuertemente regulados por las que Lillis (2013) Ilama instituciones centralizadoras. Estos programas surgidos en el ámbito académico anglosajón desconocen la singularidad de los contextos de prácticas propias de nuestras universidades.

Los textos del Módulo específico del CPU se proponen brindar información significativa acerca del plan de estudios (estructura, orientaciones, enfoques), de las características y organización del campo disciplinar, y propiciar la reflexión crítica acerca de la elección de la carrera, de las expectativas previas, de las representaciones acerca del ser profesor de lengua y literatura, del trabajo en la escuela secundaria, y de las diversas trayectorias posibles en variados contextos laborales y sociales.

Para caracterizar estos textos definimos un primer grupo compuesto por aquellos que se refieren a experiencias y trayectorias profesionales de los autores, narrados en primera persona a manera de diarios, o referidos por colegas o que son el resultado de transcripciones de entrevistas en los que se cruzan la vida personal con la vida profesional, tanto en la enseñanza secundaria como universitaria.

Entre los referidos a secundaria se incluye el diario de Juan Sánchez Enciso, un profesor español que decide ejercer la docencia en una escuela marginal en lugar de desarrollar su carrera en la universidad o "Una vida de mierda", entrevista incluida en el libro colectivo coordinado por Pierre Bourdieu, La miseria del mundo, en la que Fanny, la profesora entrevistada, plantea tensiones entre su vida familiar y profesional. Otros ejemplos son el texto del escritor y profesor peruano Jorge Slava, "Un placer ausente. Apuntes de un profesor sobre la cultura escolar" quien reflexiona -a partir de cierto encanto y cierto desencanto- acerca de la tarea docente, y el de Walter Lezcano, "Partes de guerra docente en el deep conurbano", que narra los avatares administrativos de un docente que inicia su camino profesional en la enseñanza pública. Entre los referidos al ámbito universitario se cuentan el texto de Enrique Pezzoni, "Imagen de
Ana María Barrenechea", en el que el reconocido crítico literario expone la profusa trayectoria de su consagrada maestra, y en diálogo con este texto se incluye una entrevista a la propia Barrenechea. A manera de espejo, se da a leer un texto en el que Jorge Panesi homenajea a su maestro, el profesor de literatura Enrique Pezzoni. Completa el grupo fragmentos del libro Letras muertas. De la enseñanza de las letras en general y de la cultura general en particular, de la profesora universitaria francesa Daniele Sallenave, que con un dejo de nostalgia evoca su formación literaria y cultural en los años 1960 para reflexionar acerca de la situación actual de sus estudiantes.

Otro agrupamiento incluye textos sociológicos, pedagógicos y didácticos referidos a la enseñanza de la lengua y de la literatura en el ámbito formal y no formal de la educación. Entre ellos se encuentran el artículo de Henry Giroux, "Los profesores como intelectuales transformativos", de Ramón Flecha, un capítulo de su libro Compartiendo palabras, "Lola, de 'analfabeta' a creadora de la tertulia literaria", y la entrevista a Pierre Bourdieu en "Lo que quiere decir hablar". También se incluyen textos de los propios profesores del módulo: "Conflictos del profesor", del libro Lenguas propias/ lenguas ajenas, de Paola Iturrioz, y "Vidas de profesores", del libro Reinventar la enseñanza de la lengua y la literatura, de Gustavo Bombini.

Por el último, el grupo de los textos ficcionales incluye cuentos de Martín Kohan, "Lengua y literatura", y de Roberto Fontanarrosa, "Maestras argentinas. Clara Dezcurra", que parodian aspectos y escenas de la vida escolar.

Las producciones aquí analizadas son el resultado de la actividad final del Módulo específico. La consigna solicitada para su realización domiciliaria fue enunciada de la siguiente manera:

El texto "Ana María Barrenechea" de Enrique Pezzoni es un claro reconocimiento a la trayectoria académica, profesional y personal de "Anita": sus enseñanzas, sus viajes, sus preocupaciones, su historia personal, las anécdotas; en fin, el recorrido vital que, 
como sucede cuando admiramos a alguien, siempre está teñido del afecto y de una mirada complaciente o crítica. Le pedimos que tome la voz de un "supuesto" discípulo suyo y escriba un texto de homenaje a usted. Imagine que será leído en el Congreso sobre la enseñanza de la lengua y la literatura "Homenaje a ..." (complete con su nombre y apellido) a realizarse en la UNSAM en el año 2073. Dos carillas serán suficientes para narrar sus virtudes y su trayectoria.

Seguramente el género homenaje no suele formar parte del repertorio de textos habitualmente leídos o propuestos para la producción en el espacio académico. Sin embargo, y considerando el concepto zona de pasaje, ya definido como un tiempo "entre lo conocido -la escuela secundaria y otras experiencias culturales diversas- y lo nuevo propuesto por los estudios universitarios. Nuevas relaciones con el conocimiento, nuevas instituciones, nuevos reglamentos, nuevas institucionalidades, nuevos textos, nuevas discusiones y modos de encararlas" (Bombini y Labeur, 2017, p. 9), sostenemos que las prácticas de lectura y escritura solicitadas requieren de una reconsideración respecto de los géneros más estandarizados. Se trata de recuperar para la propuesta didáctica la concepción bajtiniana de género discursivo como una categoría que permite reconocer el carácter histórico y cultural, así como también la riqueza y la ilimitada variedad de estos géneros, por cuanto dependen de cada esfera de uso y de los estilos personales y repertorios particulares. Dentro de esta concepción de la actividad discursiva (ya presentada en el marco teórico) retomamos la categoría de "géneros de enseñanza" (Rockwell, 2018, p. 575), pues nos ha permitido la multiplicidad de géneros para la lectura y la producción en el espacio del curso de ingreso aquí analizado.

Desde esta perspectiva bajtiniana, en la formulación de la tarea de escritura solicitada en el módulo, decidimos atender a la situación de zona de pasaje, evitando la exigencia de un dominio inmediato de las convenciones retóricas de los géneros propios de la escolarización académica a favor de una secuenciación que permita la paulatina construcción de disposiciones favorables hacia la escritura en el ámbito universitario, la producción de significados e identidades. En definitiva, se trató de una decisión pedagógica sostenida a partir del año 2010 en la UNSAM, que entra en diálogo con preocupaciones planteadas por la investigadora Theresa Lillis (2013) quien, en la cita que sigue, recupera las consideraciones de English acerca del uso de los géneros y su impacto:

Si bien hay una agencia evidente en la recontextualización activa de prácticas altamente reguladas, actualmente también hay intentos de abrir los espacios semióticos altamente regulados a una gama más amplia de creación de significados y, por lo tanto, a oportunidades más diversas para la producción de identidad. Centrándose en la escritura en la educación superior, por ejemplo, English argumenta que es necesario repensar el género como un recurso creativo. Los géneros orientan a las personas no solo hacia la producción de determinados textos sino hacia determinadas identidades discursivas $y$, argumenta English, una vez que el "género" se reconceptualiza como un recurso, los escritores estudiantes pueden ser invitados a explorar y jugar con una variedad de formas de producción de sentido, y de identidad, en la academia. English dice: "Los estudios de géneros han tendido a centrarse en cómo parecen ser los géneros (propiedades estructurales) o para qué se usan (enfoques funcionales) mientras que en mi estudio he tratado de centrarme en qué es lo que el género nos permite hacer" (English 2011, p. 200). (Traducción de las editoras). (p. 126)

La escritura del homenaje, en cuanto género que invita a la exploración y el juego, al decir de English, supone la recuperación de las lecturas mencionadas inicialmente junto con la memoria y el eventual registro a través de notas de clase de las discusiones mantenidas con los profesores y compañeros a lo largo de los seis encuentros, entramados con las decisiones singulares que cada estudiante pone en juego a la hora de proyectar, 
imaginar, explorar en clave de deseo, de prácticas sociales y discursivas transitadas y por transitar, su futuro profesional y personal como aspectos significativos de la construcción identitaria.

La decisión pedagógica del trabajo con el género homenaje no supone centrar la atención en aspectos estructurales y funcionales propios de su caracterización como una convención estable, sino que en el requerimiento pragmático de imaginar un texto que deberá ser dicho en voz alta frente a un auditorio, se busca discutir la división dicotómica habitual entre oralidad y escritura (Vich y Zavala, 2004), lo que habilita entonces la presencia en los textos de voces propias de sus comunidades discursivas y la renegociación de significados de voces ajenas, tomadas de los textos leídos en el módulo y otros propios de sus trayectorias textuales.

Desde otro campo disciplinar, relevante en la construcción de una propuesta didáctica inevitablemente interdisciplinaria, recuperamos la idea de creación narrativa del yo del psicólogo cultural Jerome Bruner (2002):

Comenzaré afirmando resueltamente que en efecto no es dado a conocer un yo intuitivamente evidente y esencial, que aguarde plácidamente ser representado con palabras. Más bien, nosotros construimos y reconstruimos continuamente un $\mathrm{Yo}$, según lo requieran las situaciones que encontramos, con la guía de nuestros recuerdos del pasado y nuestras experiencias y miedos para el futuro. Hablar de nosotros a nosotros mismos es como inventar un relato acerca de quién y qué somos, qué sucedió y por qué hacemos lo que estamos haciendo. (p. 19)

En esta construcción narrativa del yo, los estudiantes despliegan diversidades de escenarios y de prácticas que van desde la más previsible y reconocible trayectoria como profesor de la escuela secundaria, hasta los insospechados modos de circulación de la cultura escrita en el oficio profesional de una editora del futuro que organiza un museo literario "en el cual mediante hologramas se trajeran a la vida ciudades ficticias y personajes históricos".

A la vez, estas narrativas del yo se muestran atravesadas por avatares, obstáculos, hechos fortuitos vinculados con la vida familiar, con circunstancias políticas, con emergentes sociales y de salud pública.

En este sentido, estos relatos parecen poner en evidencia la decisión que toman sus autores de dar cuenta de sus identidades de origen, de comunidades de prácticas (Lillis, 2013); de ahí la presencia en muchas de las producciones de narraciones de infancia, de referencias a las constituciones familiares y a prácticas culturales vernáculas: "Creció rodeada de cumbia, los vinos los domingos e historias antes de ir a dormir"; "Nació, creció y vivió toda su vida rodeada de su barrio, de chica jugando a las escondidas y andando en bicicleta"; "los días yendo a vender ropa a alguna feria americana para hacerse unos mangos $y$, también, a veces, los cientos de peleas y violencia".

Si bien se trata de una propuesta que los invita a pensarse en clave de una identidad profesional futura, es destacable la necesidad de explicitar sus pertenencias de clase a la manera de una cierta lealtad a su grupo de referencia contra una representación según la cual la posesión de conocimientos académicos y la obtención de títulos implicarían un distanciamiento de ese mundo de origen y sus valores. Así, respecto de dos homenajeados se dice: "No dejaba que su título se le subiera, de alguna forma, a la cabeza" y "Fue una persona humilde que nunca tuvo aires de grandeza ni mucho menos".

La necesidad de reafirmar ese yo esencial de origen, que en ningún caso da cuenta de marcas de pertenencia a la clase alta ni de vidas acomodadas, pareciera querer mostrar el desafío que ellos consideran se les impone en el futuro: combinar los valores del presente con las proyecciones de sus vidas futuras.

Al respecto, estas operaciones realizadas en los textos ratifican las teorías del sentido común que 
rigen la producción de relatos biográficos y autobiográficos, tal como lo expone Bourdieu (1997), en los que la vida se narra como un camino, un paso con sus encrucijadas, un viaje, un desplazamiento lineal y unidireccional bajo el supuesto de que la vida constituye un todo coherente y orientado, con un orden lógico que es lo que le da sentido a la experiencia narrada.

Si bien los homenajes parecen ajustarse a los cánones de la narrativa biográfica, la lectura y el análisis de estos textos desafían al lector (los profesores que los solicitan, los autores y los lectores de este artículo) a reconocer la singularidad sociocultural de las voces que se manifiestan en estas escrituras asumiendo el desafío planteado; en términos de Bourdieu, de "aprehender la singularidad en el relato totalitario y coherente", lo que necesariamente tiene una consecuencia pedagógica, institucional y política que es la de estar dispuestos a reconocer quién es el sujeto ingresante en clave de su construcción identitaria, a partir de sus marcas de origen, sus intereses, deseos, gustos y modos de decirlos. Es quizá el género solicitado en la consigna el que mejor combina lo retrospectivo en tanto lo dado y lo prospectivo entendido como lo deseado, proyectado, imaginado, acaso lo utópico.

Avanzando hacia una posición más político-pedagógica sobre la práctica de escritura en la universidad y su relación con lo teórico interesa esta cita del pedagogo crítico norteamericano Henry Giroux:

[...] la lectura y la escritura ofrecen a los estudiantes la oportunidad de reescribir los textos de la cultura dominante, cargándolos no simplemente de sus propias experiencias, sino de una conciencia teórica perfectamente afinada, pues es en el proceso de escribir donde se dan las posibilidades de que los alumnos entiendan cómo están inmersos en el lenguaje, qué significa volver a escribir el lenguaje como acto político de compromiso crítico, y aprendan cómo escribir en diversas formas de alfabetización. (p. 192)

\section{Los valores resaltados}

A la vez, como correlato de lo anterior, se advierte la necesidad de enfatizar acerca de los valores que se sostienen en las narrativas de homenaje como inclaudicables, pese a los nuevos posicionamientos sociales propios de un recorrido académico y profesional exitoso. De este modo expresan: "Debo destacar que su humildad se ve reflejada en su trabajo", "Nos trataba de igual", "Es empática y puede entender situaciones", "Enseñaba de una manera humilde y sabia", "En su generosidad llegaba a todo el mundo que lo necesitara".

La necesidad de destacar la elección de estos valores parece reconocer como contrapartida una representación negativa acerca del ser académico, opuesta a los valores que ellos defienden:

Siempre ha sido una persona muy generosa y cálida. A lo largo de mi vida he compartido muchos momentos con otros profesores con mucha experiencia o jefes de cátedra y muchos de ellos tenían actitudes soberbias, en cambio Ignacio nunca demostró ser arrogante. Incluso era más humilde que otros profesores que recién arrancaban. Sin duda gracias a su sencillez los alumnos y alumnas lo veían tan cercano y siempre lo han querido muchísimo.

En sus narrativas, algunos estudiantes clasifican el mundo académico y suponen que los mejores calificados son los mejores clasificadores de la estructura escolar; en términos de Pierre Bourdieu y Monique Saint-Martin (1998) en "Las categorías del juicio profesoral", los sociólogos muestran que las mejores ubicaciones en el campo académico o escolar, avaladas por las credenciales y los títulos, determinan la reproducción de la estructura social. En los relatos nombran al jefe de cátedra, al director de la carrera, al discípulo, al que "recién arranca" y, por oposición, se proyectan de otra manera pues esos posicionamientos son dados vueltas: el jefe de cátedra es generoso, el autor consagrado se dedica a ir a los talleres barriales, el 
director de la carrera viaja a las provincias a modificar planes de estudio. Por un lado, como dijimos, pareciera que la insistencia en los relatos que resaltan las marcas de origen daría cuenta de cierto temor al desdibujamiento de la propia identidad a causa de la obtención de las credenciales académicas y docentes. Pero también daría cuenta del interés por modificar la desigual e injusta estructura social y revertir los destinos asignados de antemano de quienes serán sus futuros alumnos, de los participantes en talleres barriales, de los asistentes a la biblioteca popular "como un refugio", en ese barrio marginal "que tiene gusto a lo nuestro".

En esta línea, muchos de los textos imaginan que, para revertir las situaciones de exclusión y la reproducción de la desigualdad, escuelas, talleres o bibliotecas cumplirían una función transformadora a mano de las intervenciones de algunos de los homenajeados que han sabido posicionarse pedagógicamente para evitar mecanismos de estigmatización frente a las potencialidades de sus alumnos: "Su lema era 'nunca digas con estos chicos no se puede'".

En clave de construcción de identidades profesionales, uno lo de los homenajeadores explica a partir de la experiencia autobiográfica el posicionamiento político-pedagógico de su homenajeada: "Había en ella una sed de reconocimiento, incluso hasta de venganza, de demostrarle a todos quienes no le tuvieron confianza que había llegado a donde quería estar".

En el modo de construir la trayectoria profesional que se reconoce en el homenaje, se puede observar un segundo momento, donde la "sed de reconocimiento" y "venganza" inicial opera como motor para el despliegue de un recorrido destacado: "Y, cuando descubrió que el encanto agridulce se había disipado, siguió por el camino que todos conocemos".

Esta toma de conciencia de las problemáticas sociales y económicas se traduce en una mirada sensible hacia las cuestiones más básicas aún irresueltas; por ejemplo, la falta de recursos para alguna actividad de apoyo escolar: "Inició con sus compañeros y compañeras un programa de apoyo escolar para quienes no pudieran afrontar costos".

$\mathrm{O}$, de mayor gravedad, la máxima sostenida por la misma homenajeada y recordada por su exalumno a través del discurso directo: "Chicos, con hambre no se puede estudiar, prefiero que comamos a que estén pensando en el morfi".

Estas tomas de posición suponen decisiones respecto de lo profesional. Por un lado, la posibilidad de asumir tareas sin recibir retribución, tal el caso de Nahuel que, desde su formación inicial hasta su consagración, asume la gratuidad como un valor: "

Nahuel decidió inscribirse en el Voluntariado UNSAM mientras estudiaba para convertirse en docente.

Entonces empezó a dar apoyo secundario gratuito por su cuenta, a jóvenes y también a adultos que estuvieran cursando la secundaria.

También, durante el tiempo que tenía disponible, trabajaba en bibliotecas populares en otras zonas del conurbano. Para poder hacerse conocido en las instituciones se promocionaba por medio de carteles y redes sociales y siempre aclarando que él no cobraba ningún tipo de cuota, ni recibía ningún sueldo de parte del Estado para hacer eso, era algo absolutamente gratuito y solidario.

Y de manera excluyente en la mayoría de los homenajeados, la opción por la escuela pública: "Esto se relaciona en gran medida con su trayectoria en colegios públicos, incluso nunca ejerció en ningún colegio privado. 'Va en contra de mis principios', repetía".

En algún sentido, los homenajeadores asumiendo sus discursos son la prueba de la eficacia de las intervenciones pedagógicas y políticas de los homenajeados, por cuanto re-enuncian las ideas de educación trasformadora, de justicia social y la conciencia clara acerca de las dimensiones sociales y económicas que atraviesan la práctica educativa. 


\section{Concepciones de lengua y literatura}

Directamente vinculado con la elección de carrera realizada y como otro modo de reconocer la construcción de identidad que se pone en juego en los textos analizados, interesa indagar acerca de las concepciones sobre lengua y literatura que sostienen los ingresantes en sus homenajes, en calidad de doble objeto de estudio y de enseñanza de la carrera de Profesorado en Letras. También, preguntarse en qué sentido estas concepciones dialogan o no con las de la escolarización previa en el nivel secundario, y qué otras ideas o representaciones provenientes de la experiencia extraescolar se ponen en juego a la hora de dar cuenta de los objetos en cuestión.

Llama la atención en la lectura de los textos analizados la ausencia de marcas de la experiencia de formación en lengua y literatura en la escuela secundaria. Para el caso de lengua no se retoman categorías de la más tradicional enseñanza de la gramática ni de los más recientes enfoques textualistas o comunicativos. Si para algo se recupera el concepto de lengua es para dar cuenta de las reivindicaciones en clave de género: "No le gustaban las discriminaciones de ningún tipo y era inclusiva en todos los sentidos. Usaba el lenguaje inclusivo desde sus inicios de profesora y era una luchadora por los derechos de la mujer".

Para el caso de la literatura, tampoco se observa, en los textos analizados, la marca propia (o al menos esperable) de la escolarización en secundaria, en la que tradicionalmente se recurre a la organización de las literaturas como literaturas nacionales (fundamentalmente española, latinoamericana y argentina) y dentro de estos recortes el reconocimiento de unos ciertos textos considerados los clásicos de cada literatura nacional. Tampoco hay referencias a ciertas lecturas también usuales en la enseñanza escolar actual rotuladas por el mercado editorial como literatura juvenil, que suelen tratar aspectos problemáticos de las experiencias adolescentes.
Caso excepcional es el de Jorge Luis Borges que no se recupera como un clásico escolar sino más bien como exponente máximo de la alta cultura, pero sometido al gran desafío que asume uno de los homenajeados: "Ese día con sus treinta y cortos años explicaba Borges para el ciudadano de a pie. Ya se ganó el título de estar entre los primeros grandes divulgadores de la literatura en español".

Esta preocupación por la accesibilidad de la literatura propia del divulgador se manifiesta también a partir de referencias a tareas realizadas por distintos homenajeados en bibliotecas populares, "lugares complicados alejados de zonas humildes de $\mathrm{CABA}$ ", en "talleres de escritura creativa con personas mayores". La literatura se imagina como una práctica social y cultural, relacionada con la divulgación y la promoción que puede darse tanto desde la participación en la gestión estatal, tal es el caso del homenajeado que Ileva adelante "El enorme Plan de Las Letras en Casa, ideado desde el Ministerio de Educación", como desde el ámbito privado participando en eventos: "También solía asistir a las famosas 'Comic Con' para realizar algunas firmas de sus respectivos libros que encajaban en el género fantástico".

Interesa señalar cierta singularidad en cuanto a las concepciones de autor que es posible leer en los textos. La primera característica que Ilama la atención es que los homenajeados, además de reconocer una trayectoria docente y académica, se presentan como escritores muy ligados a su tiempo, a los emergentes de época, como es el caso del movimiento feminista que habilita tanto la escritura de textos de ficción y de opinión como la participación activa incluso en ámbitos internacionales:

No leer hasta el día de mi muerte y La casa de al lado, historia que relata el secuestro y tortura de una joven capturada por su vecino publicada en 2030 y Cuestión de derecho, no de opinión del 2037 que explica y critica a las personas en contra de la legalización del aborto que estuvo desde el 2008, y cómo impactan los femicidios en el pueblo y además 
toda la revolución feminista a través de los años. Este último libro fue la que la hizo, como quien diría, conocida. Desató discusiones interminables que permanecen hasta el día de hoy en redes sociales de gente conservadora y progresista. Viajó a países como España, Suecia y Francia formando parte del movimiento feminista con el objetivo de promocionar su libro y presentándolo en las manifestaciones como las del 8 de marzo, Día de la Mujer. Otros libros como Cabeza de medusa, publicado en 2043 con anécdotas fantásticas del famoso personaje mitológico y Dios es mujer, un libro que recomiendo, en el que habla sobre cómo se sentía la autora tras los momentos vividos en las manifestaciones de lucha femenina y su participación a ese grupo, porque su pensamiento fue un punto de partida que le permitió llegar adonde está hoy.

Otra característica tiene ver con la elección de género literario como marca estética ligada, a su vez, a un posicionamiento en el mercado editorial entendido como parte del circuito de consagración. Muchos de los homenajeados/escritores producen sagas exitosas en algunos casos presentadas en los textos/homenaje a la manera de recomendaciones en las redes sociales o de los booktuber:

Crónicas de un Morningstar, su clásica saga cuyo contenido se prolongó por dos décadas enteras. Un clásico medioevo que se altera por una mitología y personajes verdaderamente únicos, cuya identidad se resalta desde la escena que les presenta una atmósfera que representa un temor palpable en cada capítulo.

Otro homenajeador elige preguntarle a su auditorio:

¿Qué pasará con el capítulo 9 ya anunciado antes de su muerte, que iba a salir como concusión a su saga Viajes infinitos? Pues bien, me ha dejado como pedido que yo, su discípulo, terminase la obra. La enfermedad que llevaba lo terminó de matar antes de que pudiese concluirla. De hecho, había decidido dejarme a mí la tarea inclusive antes de terminar su octava entrega.

En este caso, como en otros, vemos que se opera en contra de la idea de autoría como la de un sujeto único pues su tarea puede ser delegada por la propia fuerza narrativa de las sagas y porque se aclara que al autor-primero "no le importaba mucho la fama, el reconocimiento, menos los trofeos" ${ }^{\prime \prime}$ distanciándose así de la idea de originalidad del autor.

Los autores homenajeados parecen circular por nuevas instituciones literarias que ya no son ni el circuito escolar, ni el académico, en cuanto instancias de consagración, sino el mercado editorial globalizado o la editorial digital o la producción de libros animados.

Por fin, es necesario resaltar que a lo largo de los textos analizados se presenta como recurrente un cierto campo semántico referido a la experiencia de la lectura y su transmisión en el que abundan términos como "placer", "amor a la lectura", "cariño a los libros", "pasión por la literatura", "magia", "mundo de sensaciones", a los que podríamos leer como metáforas cristalizadas de una concepción de literatura que forma parte de una representación social que circula en el discurso escolar y social.

Como cierre de este apartado, vale la pena transcribir por su originalidad e ingenio algunos títulos de las obras de ficción, así como también referencias a sus contextos de producción y las temáticas abordadas. Entre los títulos, se incluyen: La trilogía de novelas: Dormir es una opción, En el apogeo de un sueño y En la primavera del apogeo; el libro de cuentos: Ali Peroné y las 40 ratas; la obra de teatro Cachiponpón y Prosodia en limón, en poesía. Algunas descripciones de las temáticas son: "Espuelas en mi hogar, novela que narra las monotonías del ser humano al Ilegar a una etapa de declive dentro del matrimonio y cómo las consecuencias de esto pueden convertirse en algo horrible"; y "El descamisado donde narra la historia de su bisabuelo desaparecido en la dictadura 
cívico militar de 1976, que fue reconocido por el premio Herralde de literatura en el año 2050".

Y como ejemplo de contexto de producción citamos:

No duró ni un mes en la UBA. Su psicólogo le recomendó abandonar la carrera y tomarse un año sabático casi por la fuerza, y en ese tiempo se dedicó completamente a su primera novela: El asesino de Dios. Novela que en 2023 se volvería su primer best seller a tan corta edad.

\section{Los homenajeadores}

Si lo característico del género homenaje en un Congreso Académico supone la elección de un locutor legítimo del mismo campo, para el caso de los homenajes escritos por los estudiantes, los homenajeadores en general no son figuras con trayectoria en el campo académico sino de procedencias diversas. Se trata de alumnos de talleres barriales, algún antiguo colega de la escuela secundaria, o un profesor de otra área como música que conoce a la homenajeada desde niña, un compañero de militancia, un familiar o una vecina: "Fue entonces cuando comenzó a visitar la Unidad Básica de San Martín. Ahí la conocí"; "Recuerdo cuando empezó a dar clases en nuestra escuela y yo estaba comenzando por segunda vez mi último año de la secundaria"; "Mi nombre es Ambar, Carla es mi tía y yo soy su más fiel seguidora".

Posiblemente algo de esta elección tenga que ver con la importancia asignada a los relatos de la infancia, de la vida barrial, de las primeras experiencias profesionales y también con ciertas maneras de desarrollar el trabajo en la enseñanza y la formación, con la insistencia en los valores asociados al origen (mencionados más arriba).

Si el acento en tantos relatos está puesto en la sencillez, en la humildad, la puesta en valor del trabajo en contextos complejos, en las distintas intervenciones para paliar las injusticias, todo esto explica la elección de los homenajeadores como pertenecientes al mismo horizonte social de experiencia y, por tanto, como garantía de un universo de valores compartidos. También explica la selección, en muchos casos, tanto de un registro propio del sociolecto vernáculo que se consideraría inaceptable (en términos de Bourdieu) para el género solicitado, como también cierta elección discursiva en cuanto al tono, al repertorio y a la retórica que se presume la correcta para el acto de homenaje de un profesor en el punto culminante de su trayectoria. Esta elección de una lengua que se toma prestada para la producción de los discursos considerados prestigiosos como los académicos se explica a través de lo que Blanche-Benveniste (1982) denomina lenguaje dominguero, para designar a los usos no cotidianos, a los que se recurre en situaciones especiales al modo de esa ropa que se saca a relucir los días domingo (p. 247).

\section{Recorridos profesionales múltiples y simultáneos}

La lealtad a sus identidades de origen, la idea de la literatura como una práctica social y cultural accesible, la elección de quienes son los homenajeadores posiblemente tienen como consecuencia la configuración de perfiles profesionales que se caracterizan por desarrollar múltiples tareas diversas entre sí, en muchos casos en tiempos simultáneos, algunas previas o por fuera de los estudios universitarios en combinaciones poco habituales; es el caso de una licenciada en Letras, que también es licenciada en Sociología, quien a su vez enseña danza para promover la literatura, o el caso de un escritor de sagas que "sacó ocho libros seguidos de literatura fantástica" que es a la vez docente universitario de griego y también de latín. Lo relevante es que el perfil del académico nunca se presenta como un desarrollo profesional único y sesgado por una hiperespecialización, sino combinado con otros recorridos profesionales que precisamente lo descentran de la pertenencia exclusiva al campo académico. 
Un solo texto entre los analizados presenta un perfil académico con dedicación a la investigación, pero es abordado en tono paródico:

[...] dictaba seminarios optativos a los que iba poca gente, con títulos extensos y rimbombantes ("Sobre la musicalidad y la escritura en las corrientes románticas de la poesía argentina en el siglo XX") y publicaba libros (con colegas de otras universidades) con títulos también paródicos: Genealogía del tono poético, Se respira como se escribe e investigaciones que asumen el mismo tono donde, por ejemplo, "compara la primera imagen de las festividades griegas con los carnavales latinoamericanos y se piensa la relación entre el ditirambo griego y las letras de la comparsa de una forma maravillosa, así como los ritmos del candombe y los versos yámbicos".

\section{Conclusiones}

Lo realizado hasta aquí sobre la experiencia del trabajo en el curso de ingreso tendrá un segundo momento en el que se relevarán, a través de distintos instrumentos propios del análisis etnográfico, los procesos de negociación, de resignificación, de transformación, de adaptación o resistencia, como parte del avance de cada estudiante en el recorrido de la carrera a partir del $50 \%$ de las materias cursadas. Observaciones de clases, protocolos de escritura, entrevistas a estudiantes, diarios o notas personales y producciones académicas desarrolladas para las distintas cátedras serán insumos significativos para el análisis de los procesos antes mencionados en la producción de identidades profesionales.

En síntesis, hemos partido desde el punto de vista pedagógico de la convicción acerca de la necesidad de promover a partir de la oralidad, la lectura y la escritura en el contexto del módulo, reflexiones que ponen en juego procesos de subjetivación y de construcción de las identidades profesionales asumiendo la doble complejidad que supone la narración de tipo biográfico que se juega entre lo retrospectivo, presente en las historias de origen, y lo prospectivo, en cuanto proyecto futuro.

En estos relatos, se leen de las formas más variadas, con aristas más o menos verosímiles y con distintos tonos, socializaciones familiares, barriales y comunitarias singulares, experiencias culturales y escolares previas muy distintas, representaciones que anticipan ideas y valoraciones acerca del ámbito académico, elecciones estéticas diversas, núcleos de intereses, fantasías de intervención para la transformación en los campos educativo, cultural, político y lingüístico-literario y, de manera más específica, expectativas acerca de los contenidos de la formación del Profesorado en Letras, forjadas por una generación que desarrollará su trayectoria profesional en la tercera, cuarta y quinta décadas del siglo XXI.

La siguiente cita, en la voz de un estudiante (a modo de homenaje a nuestros alumnos), parece condensar varios de los aspectos presentados en el análisis desarrollado aquí y a la vez permite entrever -creemos- futuras complejidades que habrán de transitar en el ejercicio de la profesión.

Más tarde Juan se recibió en la carrera de Ciencias Políticas y también en la Diplomatura en Relaciones Internacionales y logró mediante el uso de la Lengua unir al pueblo Argentino, cerrando así la dichosa Grieta y convirtiéndose en el primer presidente de la Argentina cuya ideología era la Peronista-No Peronista logrando captar a todos los sectores de la sociedad, construyendo un país con bases sólidas en materia económica, social y literal.

Mediante sus planes de expansión literaria logró terminar con el problema del coronavirus y también de otras enfermedades como el dengue ya que, gracias a como todo el mundo se encontraba encerrado leyendo, no se propagó el contagio de las susodichas. 


\section{Reconocimientos}

La producción de este artículo se enmarca en el proyecto de investigación "Estudiantes y docentes en los inicios de la vida universitaria: una indagación cualitativa en el contexto de la Universidad Nacional de San Martín. Escuela de Humanidades. UNSAM. PAI 2018-2020, código G153", dirigido por Viviana Mancovsky y codirigido por Stella Maris Más Rocha.

\section{Referencias bibliográficas}

Blanche-Benveniste, C. (1982). La escritura del lenguaje dominguero. En E. Ferreiro y M. Gómez Palacio (comps.), Nuevas perspectivas sobre los procesos de lectura y escritura (pp. 135-158). México: Siglo XXI.

Bombini, G. y Labeur, P. (coords.) (2017). Prólogo. En Leer y escribir en las zonas de pasaje. Articulaciones entre la escuela secundaria y el nivel superior (pp. 9-16). Buenos Aires: Editorial Biblos.

Bombini, G. e Iturrioz, P. (2017). A propósito de los materiales didácticos en propuestas para el ingreso a la Universidad. En G. Bombini y P. Labeur (coords.), Leer y escribir en las zonas de pasaje. Articulaciones entre la escuela secundaria y el nivel superior (pp. 51-59). Buenos Aires: Editorial Biblos.

Bourdieu, Pierre (1997). La ilusión biográfica. En Razones prácticas. Sobre la teoría de la acción (pp. 74 83). Barcelona: Anagrama.

Bourdieu, P. y Saint-Martin, (1998). Las categorías del juicio profesoral. Propuesta Educativa, 9(19), 4-18. Buenos Aires: Flacso.

Bruner, J. (2002). La fábrica de historias. Derecho, literatura, vida. Buenos Aires: Fondo de Cultura Económica.

Giroux, H. (1996). Placeres inquietantes. Aprendiendo la cultura popular. Barcelona: Paidós.

Kalman, J. (2003). El acceso a la cultura escrita: la participación social y la apropiación de conocimientos en eventos cotidianos de lectura y escritura. Revista Mexicana de Investigación Educativa, 8(17), 37-66.
Lillis, T. (2013). Identity, inscription and voice. En The sociolinguistics of writing (pp. 124-149). Edimburgo: Edinburgh University Press Ltd.

Rockwell, E. (2009). La experiencia etnográfica. Historia y cultura en los procesos educativos. Buenos Aires: Paidós.

Rockwell, E. (2018). Géneros de la enseñanza. En Escritos esenciales (pp. 569-598). Buenos Aires: Clacso.

Satchwell, C. y Ivanič, R. (2010). Reading and writing the self as a college student: Fluidity and ambivalence across contexts. En K. Ecclestone, G. Biesta y M. Hughes (eds.), Lost in transition? Change and becoming through the life course (pp. 53-69). Londres: Routledge Falmer.

Vich, V. y Zavala, V. (2004). Oralidad y poder: herramientas metodológicas. Buenos Aires: Norma.

Zavala, V. (2009). “¿Quién está diciendo eso?”. Literacidad académica, identidad y poder en la educación superior. En J. Kalman y B. Street (coords.), Lectura, escritura y matemáticas como prácticas sociales: diálogos desde los estudios latinoamericanos sobre cultura escrita (pp. 348-363). México: Siglo XXI, Centro de Cooperación Regional para la Educación de Adultos en América Latina y el Caribe (Crefal).

\section{Anexo. Textos leídos durante el módulo}

Blake, Cristina; Bombini, Gustavo, y Boland, Elisa (1999). "Volver a educar. El perfil del profesor en Letras". Serie Pedagógica, 3, 99-108. Buenos Aires: Universidad Nacional de La Plata.

Bombini, Gustavo (1994). "Pero... ¿quién es el profesor?". En Otras tramas. Sobre la enseñanza de la lengua y la literatura. Rosario: Homo Sapiens.

Bombini, Gustavo (2017). "Vidas de profesores". En Reinventar la enseñanza de la lengua y la literatura. Buenos Aires: El Hacedor.

Bourdieu, Pierre (1999). "Una vida de mierda", "Una doble vida", "Con una profesora de Letras de un colegio". En La miseria del mundo. Buenos Aires: Fondo de Cultura Económica.

Bourdieu, Pierre (1984). "Lo que quiere decir hablar". En Sociología y cultura. México, Grijalbo, Consejo Nacional para la Cultura y las Artes. 
Flecha, Ramón (1997). "Lola, de 'analfabeta' a creadora de la tertulia literaria". Compartiendo palabras. Barcelona: Paidós.

Fontanarrosa, Roberto (1195). "Maestras argentinas. Clara Dezcurra". En La mesa de los galanes y otros cuentos. Buenos Aires: Ediciones de la Flor.

Giroux, Henry (1990). Los profesores como intelectuales transformativos. Los profesores como intelectuales. Barcelona: Paidós.

Iturrioz, Paola (2017). Conflictos del profesor. En Lenguas propias-lenguas ajenas: conflictos en la enseñanza de la lengua. Buenos Aires: El Hacedor.

Kohan, Martín (1994). Lengua y literatura. En Muero contento. Rosario: Beatriz Viterbo.

Lezcano, Walter (2017). Partes de guerra docente en el deep conurbano. En Los actos públicos. Buenos Aires: Letras del Sur Editorial.
Panesi, Jorge (2000). Enrique Pezzoni, Profesor de Literatura. En Críticas. Buenos Aires: Editorial Norma.

Pezzoni, Enrique (1984). Imagen de Ana María Barrenechea. En Homenaje a Ana María Barrenechea. Buenos Aires: Ministerio de Educación de la Nación.

Sallenave, Danielle (1997). Letras muertas. De la enseñanza de las letras en general y de la cultura general en particular. Buenos Aires: Losada.

Sánchez Enciso, Juan (2003). Selección de capítulos de Los mejores años. Barcelona: Octaedro.

Slava, Jorge (2013). "Lección primera" y "La lectura como discernimiento". En Un placer ausente. Apuntes de un profesor sobre la cultura escolar. Lima: Universidad de Lima. 